\title{
Changes in the Thalamus in Atypical Parkinsonism Detected Using Shape Analysis and Diffusion Tensor Imaging
}

\author{
C.P. Hess, C.W. Christine, A.C. Apple, W.P. Dillon, and M.J. Aminoff
}

\begin{abstract}
BACKGROUND AND PURPOSE: The thalamus is interconnected with the nigrostriatal system and cerebral cortex and has a major role in cognitive function and sensorimotor integration. The purpose of this study was to determine how regional involvement of the thalamus differs among Parkinson disease, progressive supranuclear palsy, and corticobasal syndrome.
\end{abstract}

MATERIALS AND METHODS: Nine patients with Parkinson disease, 5 with progressive supranuclear palsy, and 6 with corticobasal syndrome underwent 3T MR imaging along with 12 matched, asymptomatic volunteers by using a protocol that included volumetric $\mathrm{T}$ and diffusion tensor imaging. Acquired data were automatically processed to delineate the margins of the motor and nonmotor thalamic nuclear groups, and measurements of ADC were calculated from the DTI data within these regions. Thalamic volume, shape, and ADC were compared across groups.

RESULTS: Thalamic volume was smaller in the progressive supranuclear palsy and corticobasal syndrome groups compared with the Parkinson disease and control groups. Shape analysis revealed that this was mainly due to the diminished size of the lateral thalamus. Overall, ADC measurements were higher in the progressive supranuclear palsy group compared with both the Parkinson disease and control groups, and anatomic subgroup analysis demonstrated that these changes were greater within the motor regions of the thalamus in progressive supranuclear palsy and corticobasal degeneration.

CONCLUSIONS: Reduced size and increased ADC disproportionately involve the lateral thalamus in progressive supranuclear palsy and corticobasal syndrome, consistent with selective neurodegeneration and atrophy in this region. Because these findings were not observed in Parkinson disease, they may be more specific markers of tau-related neurodegeneration.

ABBREVIATIONS: $C B D=$ corticobasal degeneration; $C B S=$ corticobasal syndrome; $P D=$ Parkinson disease; $\mathrm{PSP}=$ progressive supranuclear palsy; $\mathrm{VLa}=$ ventral lateral anterior; $\mathrm{VLP}=$ ventral lateral posterior

$\mathrm{T}$ he neurodegenerative movement disorders Parkinson disease (PD), progressive supranuclear palsy (PSP), and corticobasal degeneration (CBD) are distinguished postmortem by differing histologic abnormalities and regional patterns of neuropathologic changes. PSP and corticobasal syndrome (CBS) exhibit neuronal and glial cytoplasmic inclusions from accumulation of highly phosphorylated microtubule-associated tau protein, which is not

Received July 24, 2013; accepted after revision October 2.

From the Departments of Radiology and Biomedical Imaging (C.P.H., A.C.A., W.P.D.) and Neurology (C.W.C., W.P.D., M.J.A.), University of California, San Francisco, San Francisco, California.

This work was supported by the UCSF Department of Radiology and Biomedical Imaging. C.P.H. was supported in part by the GE-AUR Radiology Research Academic Fellowship.

Please address correspondence to Christopher P. Hess, MD, PhD, University of California, San Francisco, 505 Parnassus Ave, Room L-358, Box 0628, San Francisco, CA 94143-0628; e-mail: Christopher.Hess@ucsf.edu

三 Indicates article with supplemental on-line table

http://dx.doi.org/10.3174/ajnr.A3832 evident in PD. ${ }^{1-3}$ Paralleling these differences, typical clinical phenotypes help to distinguish these disorders. However, classic presentations are consistent only in advanced disease, and misdiagnosis is frequent in patients with early symptoms. ${ }^{4,5}$ The diagnosis of CBD is particularly problematic ${ }^{6}$; for this reason, the term "corticobasal syndrome" is applied in lieu of CBD to convey the fact that disorders including Alzheimer disease, certain variants of frontotemporal lobar degeneration, and prion disease can present similarly.

Various observations on MR imaging have been reported to differentiate PD, PSP, and CBD. Alterations in the shape or volume of several subcortical brain regions correlate with gross inspection of the brain in pathologically confirmed cases. ${ }^{7}$ However, such changes are subject to observer bias and are reliably found only in late disease. Unbiased approaches by using voxelbased morphometry and automated segmentation have also been applied with some success. ${ }^{8-13}$ DTI studies have revealed increased diffusivity within the superior cerebellar peduncles in 
PSP, ${ }^{14-16}$ along with more widespread changes within supratentorial white matter. ${ }^{17,18}$ A number of groups have also described alterations in deep gray matter diffusion. For example, measurements of ADC are elevated within the putamen in up to $90 \%$ of patients with atypical parkinsonism but not significantly different from controls in patients with PD. ${ }^{16,19}$

The shared anatomic involvement of the basal ganglia and other brain regions in these disorders likely contributes to their frequent clinical overlap. Few studies have focused specifically on the thalamus, which, through the nigrostriatal system and thalamocortical circuits, plays a major role in cognition and sensorimotor integration. Alterations in thalamic volume have not been shown in prior studies of CBS, but significantly lower thalamic volume has been observed in PSP compared with both patients with $\mathrm{PD}^{20}$ and controls. ${ }^{10,20}$ Diminished volume by itself does not provide insight into how separate nuclear groups within the thalamus are selectively affected, however. One study suggested that thalamic shape differs between subjects with PD and healthy elders, though these changes were challenging to interpret because there was multifocal involvement over the entire surface of the thalamus without a corresponding difference in volume. ${ }^{21}$ Using DTI, Erbetta et $\mathrm{al}^{22}$ measured higher ADC within the anterior and lateral thalami in PSP and CBS compared with controls, but their analysis was based on relatively imprecise atlas-based estimates of nuclear boundaries. ${ }^{22}$

We hypothesized that regional thalamic morphology and tissue microstructure, measured with volumetric T1-weighted MR imaging and DTI, respectively, would be different in patients with PSP and CBS compared with patients with PD and controls. Using fully automated analysis of $3 \mathrm{~T}$ T1 and DTI data, we evaluated thalamic shape and diffusion within motor and nonmotor thalamic nuclear groups and compared these measurements in patients with CBS, PSP, and PD and in healthy control subjects.

\section{MATERIALS AND METHODS Subject Recruitment}

The University of California, San Francisco Committee on Human Research approved this study. Subjects with PD $(n=9)$, PSP $(n=5)$, or CBS $(n=6)$ were prospectively recruited from the UCSF Parkinson's Disease and Movement Disorders Clinic. Two movement disorder specialists (M.J.A., C.W.C.) independently assigned a diagnosis to all patients based on history, neurologic examination, and prior response to levodopa therapy. Patients were included only if there was agreement between the 2 clinicians. Data abstracted during patient interviews included the initial symptom and laterality (if present) at disease onset, age at diagnosis, response to levodopa therapy, and the presence or absence of cognitive symptoms. Patients with PSP met the criteria for clinically probable $\mathrm{PSP}^{23}$ and patients with $\mathrm{PD}$ met the criteria for probable $\mathrm{PD},{ }^{24}$ except for one who had not been previously treated with dopaminergic medication and therefore met the criteria for possible PD. Patients diagnosed with CBS also met accepted criteria. ${ }^{25}$ Twelve healthy control subjects without any history of neurologic illness and normal neurologic examination findings were also recruited and imaged for comparison with patients.

\section{Data Acquisition}

After providing written consent, subjects were imaged on a $3 \mathrm{~T}$ Signa HDx 14.x scanner (GE Healthcare, Milwaukee, Wisconsin) with an 8-channel head coil. To delineate thalamic boundaries, 3D T1 inversion recovery spoiled gradient-echo images were acquired in the sagittal plane with 1-mm isotropic voxel resolution and $\mathrm{TR} / \mathrm{TE} / \mathrm{TI}=7.2 / 2.3 / 400 \mathrm{~ms}$, flip angle $=15^{\circ}$, matrix size $=$ $256 \times 192$, slab thickness $=1.0 \mathrm{~mm}, \mathrm{FOV}=256 \times 192 \mathrm{~mm}(\mathrm{scan}$ time 5 minutes 54 seconds). In the same session, DTI was performed at 2-mm isotropic resolution by using a single-shot axial echo-planar acquisition with $\mathrm{TR} / \mathrm{TE}=17$ seconds $/ 68.4 \mathrm{~ms}$, $\mathrm{FOV}=256 \times 256 \mathrm{~mm}$, matrix $=128 \times 128$ zero-filled to $256 \times$ 256 (scan time 8 minutes 47 seconds). Diffusion-weighting of $b=1000 \mathrm{~s} / \mathrm{mm}^{2}$ was applied along 30 noncollinear directions. A single $b=0 \mathrm{~s} / \mathrm{mm}^{2}$ image was acquired, and array spatial sensitivity encoding technique parallel imaging was used with $\mathrm{R}=2$ to reduce susceptibility artifacts. Axial T2 FLAIR images were also acquired and visually inspected to exclude significant white matter disease and lesions within the thalami such as lacunar infarctions that might interfere with volumetric analysis, tractography, or ADC quantitation. Although some subjects in each group had a few punctate foci of nonspecific white matter T2 signal, no abnormality was evident within the thalami of any subject.

\section{Thalamic Morphology}

Before morphometric analysis, T1-weighted images were corrected for multichannel coil-related nonuniformities in intensity by using vendor software on the scanner. Six-parameter rigidbody registration to the isotropic 1-mm T1-weighted version of the Montreal Neurological Institute International Consortium for Brain Mapping 152 atlas was used to obtain gross alignment of coordinate axes among subjects. Standard space masking to remove neck and eye tissue was then performed and followed by skull stripping by using the Brain Extraction Tool, included in the FSL software package (http://www.fmrib.ox.ac.uk/fsl). ${ }^{26}$

Shape analysis relied on automated thalamic parcellation within each hemisphere by using the FIRST subcortical segmentation tool in FSL. ${ }^{27}$ Thalamic volumes were calculated from the resulting binary masks. Estimated total intracranial volume was computed for each subject by using in-house software implementing the atlas-based method developed by Buckner et al. ${ }^{28}$ Differences in thalamic morphology between groups were assessed by using the heat kernel smoothing approach for structure modeling developed by Kim et al. ${ }^{29}$ This technique allows robust, point-by-point evaluation of the displacement vector fields that map the study-specific normal template for each structure of interest onto the estimated boundaries of the same structure in each subject's brain.

Two steps were implemented to calculate surface deformation data: 1) normal template construction for each thalamus, and 2) estimation of deformation fields between the normal template and individual subject surfaces. In the first step, the group-average brain volume was derived from the images of healthy controls by using the nonlinear iterative template construction tool in the Advanced Normalization Tools software package (http://software. incf.org/software/advanced-normalization-tools). ${ }^{30}$ The deformation fields warping each normal brain to the template were then applied to the binary subcortical masks from FIRST, and the 


\begin{tabular}{|c|c|c|c|c|c|}
\hline & NC & PD & PSP & CBD & $P$ Value \\
\hline No. & 12 & 9 & 5 & 6 & \\
\hline Age (yr) & $68.6 \pm 5.5$ & $66.3 \pm 7.9$ & $69.2 \pm 3.0$ & $70.5 \pm 4.8$ & .67 \\
\hline $\operatorname{Sex}(F / M)$ & $4: 8$ & $2: 7$ & $3: 2$ & 5:1 & .09 \\
\hline Disease duration (yr) & $\mathrm{N} / \mathrm{A}$ & $8.6 \pm 4.2$ & $3.8 \pm 1.4$ & $5.5 \pm 1.4$ & $.03^{\mathrm{a}}$ \\
\hline Side of main symptoms & N/A & 5 left; 4 right & N/A & 3 left; 3 right & .24 \\
\hline eTIV (L) & $1.59 \pm 0.18$ & $1.53 \pm 0.10$ & $1.43 \pm 0.27$ & $1.58 \pm 0.32$ & .24 \\
\hline
\end{tabular}

Note:-NC indicates healthy control; L, liters; NA, not applicable; eTIV, estimated total intracranial volume.

a Statistically significant $(P<.05)$.

masks were averaged in template space to obtain mean thalamic volumes. Isosurfaces were constructed from these volumes by using the marching cubes algorithm in Matlab (MathWorks, Natick, Massachusetts).

In the second step, the deformation field for warping the template to each subject's T1-weighted images was calculated by using the diffeometric shape and averaging technique with cross-correlation as the similarity metric in Advanced Normalization Tools. Because the field is defined on voxels, deformation fields were interpolated onto the mesh vertices for the surfaces obtained in the first step. For each subject, the length of the displacement vector at each point on the surface mesh was computed. Finally, the resulting displacement lengths were smoothed by using the Laplace-Beltrami eigenfunctions. ${ }^{29}$

\section{Thalamic Diffusion}

DTI data were skull-stripped and corrected for motion and eddy current artifacts by using FSL, and ADC maps were calculated by using in-house code implementing standard diffusion reconstruction. To account for the possibility of slight misregistration between the diffusion and T1-weighted data, we aligned each T1 volume with the corresponding $b=0$ diffusion volume by using linear registration in FSL with mutual information as the registration metric.

Thalamic nuclear groups corresponding to the conglomerate ventral anterior, VLa, and VLp nuclei were delineated by using the probabilistic tractography technique described by Behrens et $\mathrm{al}^{31}$ and implemented in FMRIB Diffusion toolbox in FSL (http:// www.fmrib.ox.ac.uk/fsl/fdt/index.html). Regions corresponding to primary motor, premotor, and supplementary motor area cortices were delineated as a single region of motor thalamic projections distinct from the remainder of the cortex within each hemisphere on the standard International Consortium for Brain Mapping 152 template. By first registering the template brain to each subject's T1-weighted scan, cortical ROIs were defined in each subject and used as classification targets for seed-based tractography. The resulting segmentations were used to construct motor and nonmotor masks for calculation of ADC for the entire, motor, and nonmotor thalami on each side of the brain.

\section{Statistical Analysis}

Differences in sex among groups were evaluated by using $\chi^{2}$ tests for equivalence. One-way analysis of variance was used to compare age at the time of MR imaging, disease duration (defined as the time between approximate initial symptom onset and MR imaging), and estimated total intracranial volume. Thalamic volumes were assessed for asymmetry across hemispheres in subjects with PD and CBS by using paired $t$ tests and were subsequently compared across groups by using multivariate analysis of covari- ance with age, sex, and estimated total intracranial volume as covariates. Kruskal-Wallis nonparametric tests were used to identify intergroup differences in mean thalamic ADC, with the significance threshold at $P<.05$, followed by post hoc 1-way ANOVA with unequal variances between groups in which differences were found. For all pair-wise comparisons, differences were considered significant at a false discovery rate of $q<0.05$.

The above statistical tests were performed by using R (http:// www.r-project.org/). ${ }^{32}$ The SurfStat toolbox (http://www.math. mcgill.ca/keith/surfstat/), ${ }^{33}$ a platform-designed surface mesh and volume data analysis, was used for group comparisons of thalamic shape. This package permits multivariate shape analysis with linear mixed-effects models, including multiple-comparisons correction by using random field theory.

\section{RESULTS}

\section{Subjects}

Baseline characteristics for the 4 study groups are summarized in the Table and On-line Table, respectively. There were 4 female and 8 male controls, ranging from 61.6 to 80.6 years of age. Patient age, sex, and estimated total intracranial volume were not significantly different between groups. Disease duration was longer in the PD group than in both the CBS and PSP groups $(P=.026)$.

\section{Thalamic Volume and Shape}

There was no difference in right or left thalamic volume between healthy controls and patients with PD (Fig 1A). However, both volumes were smaller in the PSP and CBS groups compared with the healthy control and PD groups. Comparisons within groups after ordering volumes by size revealed no interhemispheric asymmetry in thalamic volume within any group. This included $\mathrm{PD}$ and CBS groups, in which asymmetric symptom onset was noted in all patients.

Using multivariate analysis with age and estimated total intracranial volume as covariates, while correcting for multiple comparisons, the study was not adequately powered to detect changes in thalamic shape specific to each group. However, on the basis of the statistical equivalence of the volumes in the CBS and PSP groups and on previously noted neuropathologic overlap between the disorders, these 2 groups were pooled for shape analysis. Figure $1 B$ illustrates the results of this comparison for the 3 groups (healthy control, PD, and CBS+PSP). There were no differences in shape on the medial aspect of the thalamus. However, differences were present bilaterally for the group with atypical parkinsonism in both the ventral anterior and ventral lateral thalamus, corresponding to the location of the thalamic motor nuclei (Fig 2). The lateral surface of the conglomerate motor nuclei was involved when considering all voxels with a trend $(P<.25)$ toward significance. 
ENTIRE THALAMUS
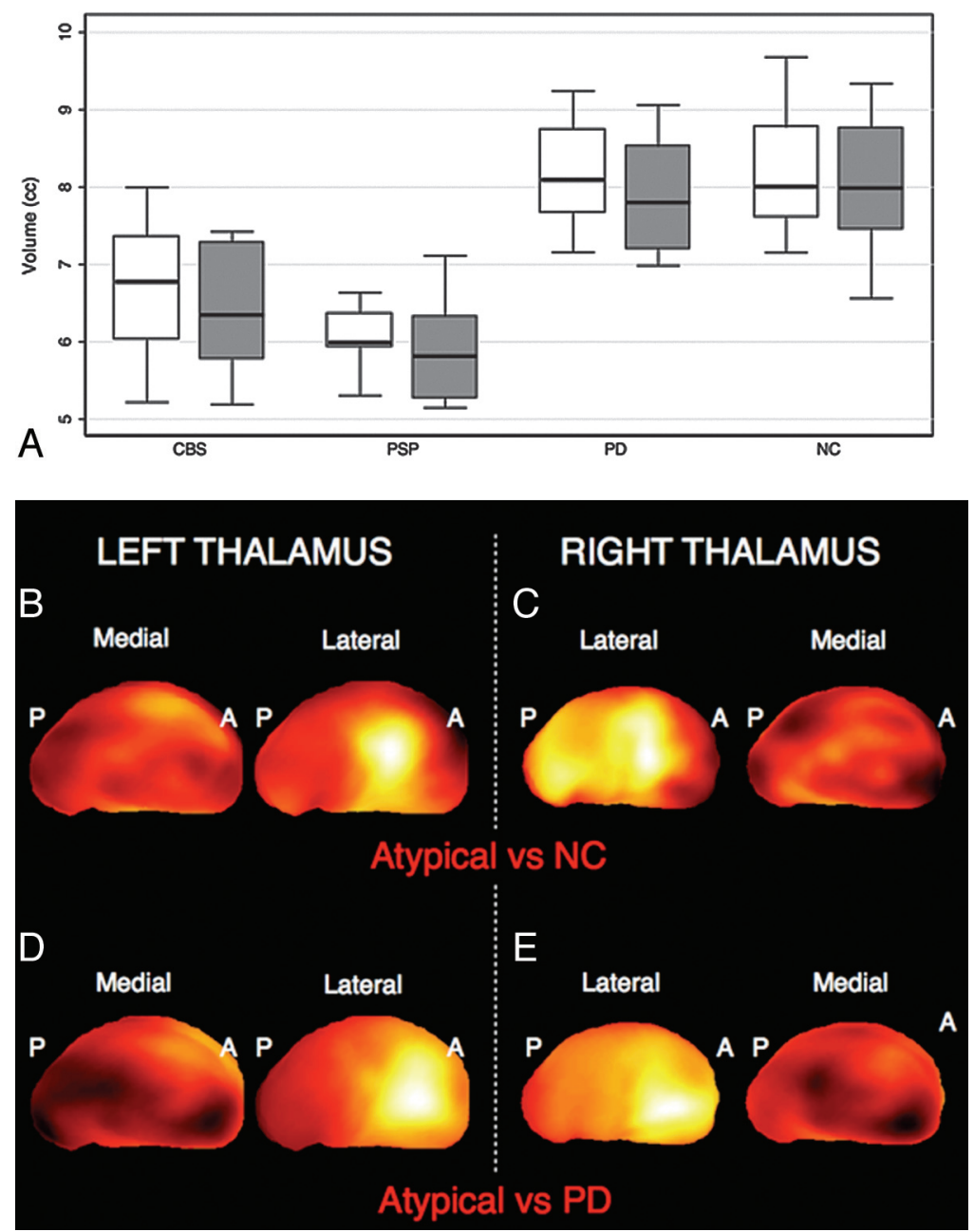

FIG 1. A, Boxplots of left (white) and right (gray) thalamic volumes in milliliters in healthy controls and patients with PD, CBS, and PSP. Vertical lines illustrate the range in calculated volume for each group, and upper and lower box boundaries correspond to 75th and 25th percentiles for volumes, respectively. Dark horizontal lines represent median values. $B-E$, Statistical maps of shape difference displayed on the average left $(B$ and $D$ ) and right $(C$ and $E)$ thalami. Note relatively the symmetric shape difference in the lateral thalamus, with relative sparing of the medial thalamus. Only white met statistical significance. Yellow indicates areas with trends toward significance $(P<.25)$, seen clustered more extensively around regions in the expected location of the ventral anterior and ventral lateral thalamic nuclei. A similar pattern of difference in shape is observed when the atypical group (CBD+PSP) is compared with the healthy control group ( $B$ and $C)$ and patients with $\operatorname{PD}(D$ and $E)$.

\section{Thalamic Mean Diffusivity}

Mean ADC values within the right and left thalami are shown in Fig $3 A$. Because there was no interhemispheric asymmetry in $\mathrm{ADC}$ values in any group, ADCs for the left and right thalami were averaged in each patient. Group differences were detected in mean $\operatorname{ADC}(P=.037)$ across the 4 groups (healthy control, PD, CBS, PSP), and post hoc between-group ANOVAs revealed differences in ADC between the PSP and healthy control groups $(P=.033)$ and between the PSP and PD groups $(P=.0081)$. Pair-wise comparisons between other groups showed no additional differences.

For analysis of ADC within thalamic nuclei, the CBS and PSP groups were combined, in analogy with the pooling of subjects used for volumetric analysis. No interhemispheric asymmetry in ADC was observed, so values were averaged for these regions in all
3 groups (healthy control, $\mathrm{PD}$, and CBS + PSP). There was no difference in ADC within nonmotor thalamic regions $(P=.31)$, but the ADC values differed within the motor thalamic regions $(P<.001$, Fig 3B). Post hoc pair-wise ANOVAs showed that motor thalamic ADC in the CBS+PSP group was significantly greater than the motor thalamic ADC in both the healthy control and PD groups $(P<.001)$.

\section{DISCUSSION}

Our results show that patients diagnosed with the atypical parkinsonian disorders CBS and PSP exhibit not only global differences in thalamic volume but also localized alterations in shape and ADC within thalamic motor nuclear regions (ventral anterior, VLa, and VLp) compared with patients with PD and controls. These changes are spatially concordant, supporting the concept of disproportionate motor thalamus involvement in these 2 disorders. Furthermore, similar changes were not found in patients with PD; this finding suggests that the differences may be specific to tau neurodegeneration. These results provide imaging evidence for alterations in thalamic substructure in atypical parkinsonism and corroborate existing histopathologic data showing motor thalamus degeneration in postmortem-confirmed cases of these disorders. ${ }^{1-3,6}$

In contrast to most prior studies that included the thalamus, we used fully automated analysis of images from a single 3T MR imaging scanner instead of manual region-of-interest analysis of $1.5 \mathrm{~T} \mathrm{~T} 1$ and diffusion data. Because the analysis was performed in the native measurement space for each subject, the methods are inherently more sensitive than voxel-based morphometry; registration of subjects to a common template and spatial smoothing to reduce misregistration errors in voxel-based morphometry can obscure group differences within small structures like the thalamus. This limitation may account for the absence of regional thalamic changes in prior studies using voxel-based morphometry.

Our work differs in several respects from the related work of Saini et $\mathrm{al},{ }^{13}$ who applied a different technique for shape analysis in a number of subcortical structures in PSP, including the thalamus. First, nonlinear registration in this work provided more precise estimates for deformation at each surface point, thereby allowing vertex-wise comparison without multivariate Gaussian assumptions. Second, the random field technique for multiple comparisons provides a smoother and more robust approach (on the mesh surface) than the false discovery rate method. Finally, changes in morphology were studied together with changes in diffusivity, helping to confirm the veracity of spatially concordant changes in both thalamic tissue microstructure and shape. 


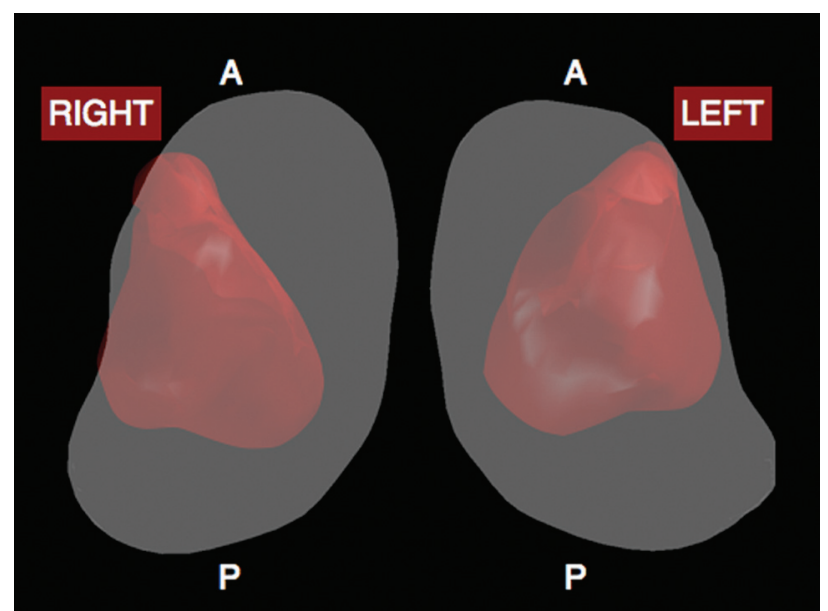

FIG 2. Correspondence between thalamic surface templates and DTI estimates of the location of thalamic nuclei, as viewed from a level superior to the thalami. The right and left thalamic motor nuclear groups, parcellated from the nonmotor regions of the thalamus by using probabilistic DTI tractography, are depicted in red. The contours of the thalami, shaded gray, were delineated automatically from the T1-weighted anatomic images. A indicates anterior; P, posterior.

\section{ENTIRE THALAMUS}

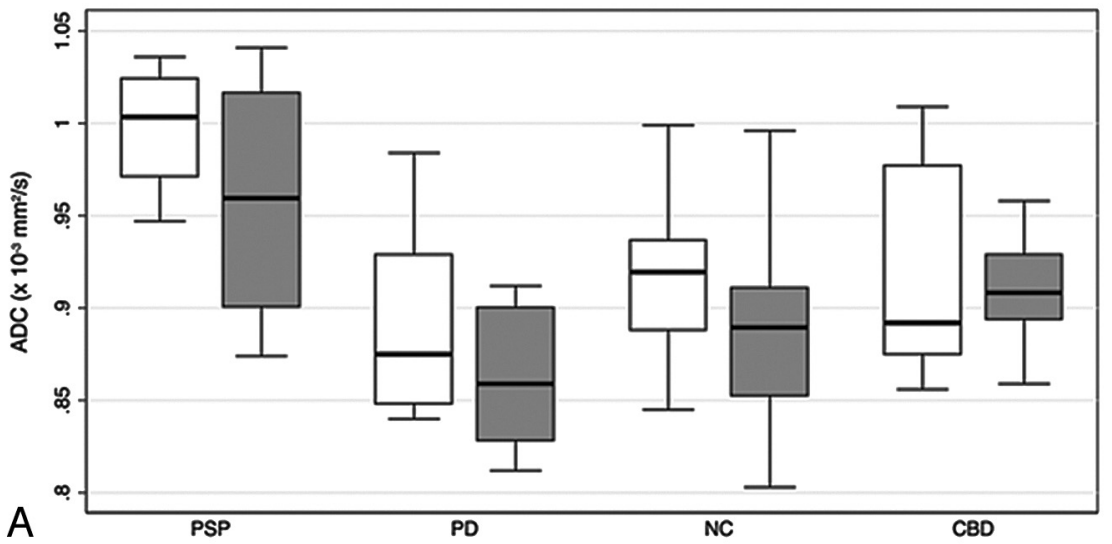

MOTOR VS NON-MOTOR THALAMIC NUCLEI

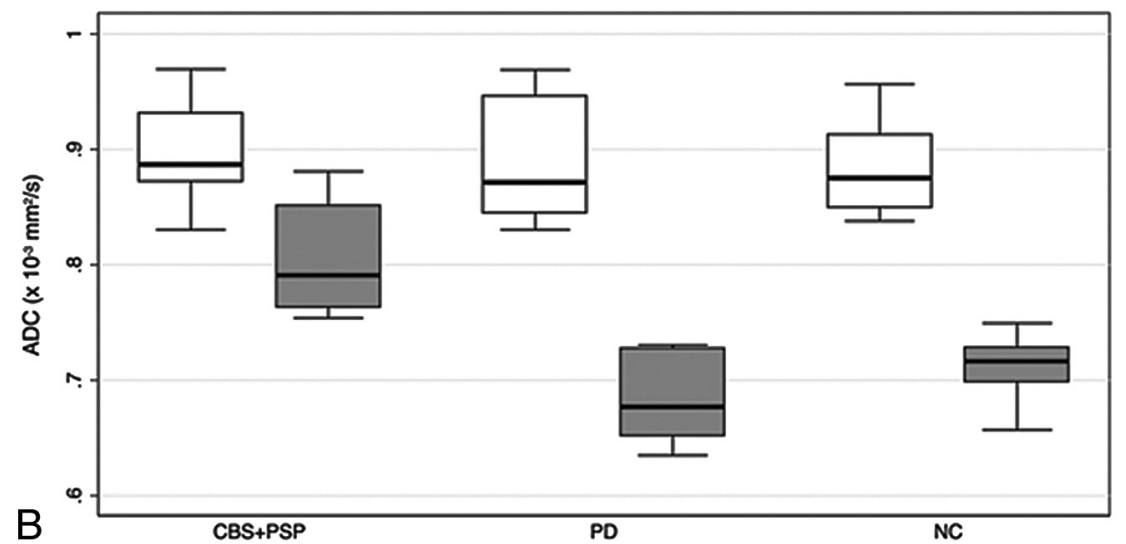

FIG 3. $A$, Boxplots of left (white) and right (gray) thalamic ADC in $\mathrm{mm}^{2} / \mathrm{s}\left(\times 10^{-3}\right)$ in control, PD, CBS, and PSP groups. Nonparametric tests of intergroup equivalence demonstrate significant differences in thalamic ADC in the PSP group compared with both healthy controls and patients with PD (see text). B, Boxplots of mean ADC in $\mathrm{mm}^{2} / \mathrm{s}\left(\times 10^{-3}\right)$ within nonmotor (white) and motor (gray) thalamic nuclei in healthy controls and patients with PD and atypical PD (CBS+PSP).
In this study, the most striking differences in thalamic shape and ADC were evident in the PSP group, and a more intermediate difference was found in the CBS group (Figs 1 and 3). This intermediate effect suggests greater disease heterogeneity in the CBS group because patients with CBS due to Alzheimer disease or other pathologies may exhibit different degrees of thalamic injury than those with tau pathology. Pathologic confirmation of diagnosis was lacking in our study, however, making it difficult to discriminate among the disorders with absolute certainty. Although it was necessary to combine patients with CBS and PSP into a single group to improve statistical power, we justify this approach on the basis of the pathologic overlap between PSP and many cases of CBS, especially with respect to tau deposition within the thalamus. ${ }^{1}$

Neuropathologic studies have shown thalamic atrophy in both CBS and PSP. ${ }^{2,3}$ Although thalamic involvement has also been described in the caudal intralaminar nuclei in $\mathrm{PD},{ }^{34}$ the central location of these nuclei makes their selective atrophy difficult to detect by using surface morphometry as applied in this study. In the absence of longitudinal data and pathology, is it impossible to distinguish whether the differences that we observed in the motor thalamus reflect primary neurodegeneration in this region or secondary "downstream" degeneration related to abnormalities arising in the basal ganglia or cortical gray matter and secondarily involving the thalamus.

Selective involvement of thalamic motor nuclei in CBS and PSP is consistent with disruption of normal thalamocortical motor connectivity. The primary motor cortex receives thalamic input from the VLp nucleus, and the ventral anterior and VLa regions are more broadly connected to the premotor cortex and supplementary motor areas. The anatomic interruption of these pathways has been associated with dystonia, alien hand syndrome, apraxia, and other clinical features evident in both CBS and PSP. ${ }^{35}$ The observation of involvement of frontoparietal white matter by using hypothesisfree whole-brain analysis of ADC in patients with $\mathrm{PSP}^{18}$ further corroborates the idea that PSP preferentially affects these pathways. Along similar lines, Whitwell et $\mathrm{al}^{36}$ used fMRI, DTI, and voxel-based morphometry to support their hypothesis that PSP causes disruption of the long, polysynaptic dentatorubrothalamic tract. The results of our work support this concept and further suggest that the overlapping phenotypes of CBS and PSP might reflect selective involvement of different segments of this pathway, with both disorders involving the motor thalamus, but with cerebellar disconnection predominating in PSP and cortical disconnection predominating in CBS. 
The present work has several limitations. First, the number of subjects was small, necessitating the combination of the PSP and CBS groups for our statistical analyses. There was a difference in disease duration between groups, and this could be relevant if the observed higher values of ADC in PSP or CBS later normalize in the disease course. Third, the boundary estimates for the thalamic nuclei derived by using probabilistic tractography are inherently imprecise, and as such, the derived ADC values may have been subject to partial volume effects between nuclear groups. Finally, we used clinical criteria for diagnosis and did not have pathologic confirmation.

\section{CONCLUSIONS}

Reduced size and increased ADC are disproportionately found in the motor thalamus in the atypical parkinsonian disorders PSP and CBS, consistent with selective neurodegeneration in these regions. The absence of similar changes in PD suggests that these differences may be specific to tau-related neurodegeneration.

\section{ACKNOWLEDGMENTS}

The authors are grateful to Seung-Goo Kim for helpful discussions regarding the implementation of deformation tensor morphometry.

Disclosures: Christopher Hess—RELATED: Grant: GE Radiology Research Academic Fellowship,* Comments: salary support, UNRELATED: Consultancy: Imaging Endpoints Inc, Comments: clinical trial consulting, Expert Testimony: medicolegal, Grants/Grants Pending: GE.* Chadwick W. Christine-UNRELATED: Consultancy: Transcept Pharmaceuticals, Comments: consulting for new Parkinson disease drug development, Grants/Grants Pending: Kinemed Inc, ${ }^{*}$ Comments: support of an ongoing biomarker study for Parkinson disease, Other: National Institutes of Health/ National Institute of Neurological Disorders and Stroke, ${ }^{*}$ Comments: support of ongoing clinical studies, in neuroprotection in Parkinson Disease. William P. DillonUNRELATED: Expert Testimony: medicolegal. Michael J. Aminoff-UNRELATED: Grants/Grants Pending: National Parkinson's Foundation, ${ }^{*}$ National Institutes of Health, ${ }^{*}$ Fox Foundation, ${ }^{\star}$ Comments: I direct the UCSF Parkinson's Disease Center, which is a National Parkinson Foundation Center of Excellence. I also have a grant through the Parkinson's Study Group and the National Institutes of Health to participate in a multicenter study of creatine in slowing the course of Parkinson disease. Finally, I have a grant funded by the Michael J. Fox Foundation to study CSF in Parkinson disease. None of this is related to the present work, Royalties: Elsevier, McGraw Hill, Oxford University Press, Wolters Kluwer Health, Comments: for books on neurology or biography. No relation whatsoever to the present work, Payment for Development of Educational Presentations: TEVA Corp, Comments: to help support an annual course on Parkinson disease for patients and caregivers. No relation to the present work. ${ }^{\star}$ Money paid to the institution.

\section{REFERENCES}

1. Feany MB, Dickson DW. Widespread cytoskeletal pathology characterizes corticobasal degeneration. Am J Pathol 1995;146:1388-96

2. Dickson DW. Neuropathologic differentiation of progressive supranuclear palsy and corticobasal degeneration. J Neurol 1999; 246 (suppl 2):II6-15

3. Dickson DW, Berferon C, Chin SS, et al. Office of Rare Diseases neuropathologic criteria for corticobasal degeneration syndrome from progressive supranuclear palsy. J Neuropathol Exp Neurol 2002;61:935-46

4. Litvan I, Grimes, DA, Lang AE, et al. Clinical features differentiating patients with postmortem confirmed progressive supranuclear palsy and corticobasal degeneration. J Neurol 1999;246(suppl 2):II1-5

5. Scaravilli T, Tolosa E, Ferrer I. Progressive supranuclear palsy and corticobasal degeneration: lumping versus splitting. Mov Disord 2005;20:S21-28

6. Ling H, O'Sullivan SS, Holton JL, et al. Does corticobasal degeneration exist? A clinicopathological re-evaluation. Brain 2010;133: 2045-57
7. Savoiardo M. Differential diagnosis of Parkinson's disease and atypical parkinsonian disorders by magnetic resonance imaging. Neurol Sci 2003;24(suppl 1):S35-37

8. Gröschel K, Hauser T-K, Luft A, et al. Magnetic resonance imagingbased volumetry differentiates progressive supranuclear palsy from corticobasal degeneration. Neuroimage 2004;21:714-24

9. Price S, Paviour D, Scahill R, et al. Voxel-based morphometry detects patterns of atrophy that help differentiate progressive supranuclear palsy and Parkinson's disease. Neuroimage 2004;23:663-69

10. Boxer AL, Geschwind MD, Belfor N, et al. Patterns of brain atrophy that differentiate corticobasal degeneration syndrome from progressive supranuclear palsy. Arch Neurol 2006;63:81-86

11. Josephs KA, Whitwell JL, Dickson DW, et al. Voxel-based morphometry in autopsy proven PSP and CBD. Neurobiol Aging 2008;29: $280-89$

12. Whitwell JL, Jack CR, Boeve BF, et al. Imaging correlates of pathology in corticobasal syndrome. Neurology 2010;75:1879-87

13. Saini J, Bagepally BS, Sandya M, et al. Subcortical structures in progressive supranuclear palsy: vertex-based analysis. Eur J Neurol 2013;20:493-501

14. Blain CR, Barker GJ, Jarosz JM, et al. Measuring brain stem and cerebellar damage in parkinsonian syndromes using diffusion tensor MRI. Neurology 2006;67:2199-205

15. Nicoletti G, Tonon C, Lodi R, et al. Apparent diffusion coefficient of the superior cerebellar peduncle differentiates progressive supranuclear palsy from Parkinson's disease. Mov Disord 2008;23:2370-76

16. Rizzo G, Martinelli P, Manners D, et al. Diffusion-weighted brain imaging study of patients with clinical diagnosis of corticobasal degeneration, progressive supranuclear palsy and Parkinson's disease. Brain 2008;131:2690-700

17. Knake S, Belke N, Menzler K, et al. In vivo demonstration of microstructural brain pathology in progressive supranuclear palsy: a DTI study using TBSS. Mov Disord 2010;25:1232-38

18. Saini J, Bagepally BS, Sandhya M, et al. In vivo evaluation of white matter pathology in patients of progressive supranuclear palsy using TBSS. Neuroradiology 2012;54:771-80

19. Seppi K, Schocke MF, Esterhammer R, et al. Diffusion-weighted imaging discriminates progressive supranuclear palsy from PD, but not from the Parkinson variant of multiple system atrophy. Neurology 2003;60:922-27

20. Messina D, Cerasa A, Condino F, et al. Patterns of brain atrophy in Parkinson's disease, progressive supranuclear palsy and multiple system atrophy. Parkinsonism Relat Disord 2011;17:172-76

21. McKeown MJ, Uthama A, Abugharbieh R, et al. Shape (but not volume) changes in the thalami in Parkinson disease. BMC Neurol 2008;8:8

22. Erbetta A, Mandelli ML, Savoiardo, et al. Diffusion tensor imaging shows different topographic involvement of the thalamus in progressive supranuclear palsy and corticobasal degeneration. AJNR Am J Neuroradiol 2009;30: 1482-87

23. Litvan I, Agid Y, Calne D, et al. Clinical research criteria for the diagnosis of progressive supranuclear palsy (Steele-RichardsonOlszewski syndrome): report of the NINDS-SPSP international workshop. Neurology 1996;47:1-9

24. Gelb DJ, Oliver E, Gilman S. Diagnostic criteria for Parkinson's disease. Arch Neurol 1999;56:33-39

25. Riley DE, Lang AE. Clinical diagnostic criteria. Adv Neurol 2000;82:29-34

26. Jenkinson M, Beckmann CF, Behrens TE, et al. FSL. Neuroimage 2012;62:782-90

27. Patenaude B, Smith SM, Kennedy D, et al. A Bayesian model of shape and appearance for subcortical brain. Neuroimage 2011;56:907-22

28. Buckner RL, Head D, Parker J, et al. A unified approach for morphometric and functional data analysis in young, old, and demented adults using automated atlas-based head size normalization: reliability and validation against manual measurement of total intracranial volume. Neuroimage 2004;23:724-38

29. Kim SG, Chung MK, Schaefer SM, et al. Sparse shape representation using the Laplace-Beltrami eigenfunctions and its application to 
modeling subcortical structures. Proc Workshop Math Methods Biomed Image Analysis 2012:25-32

30. Avants BB, Tustison NJ, Song G, et al. A reproducible evaluation of ANTs similarity metric performance in brain image registration. Neuroimage 2011;54:2033-44

31. Behrens TE, Johansen-Berg H, Woolrich MW, et al. Non-invasive mapping of connections between human thalamus and cortex using diffusion imaging. Nat Neurosci 2003;6:750-57

32. R Development Core Team. R: A Language and Environment for Statistical Computing. Vienna, Austria: R Foundation for Statistical Computing; 2005

33. Worsley KJ. A Matlab toolbox for the statistical analysis of univariate and multivariate surface and volumetric data using linear mixed effects models and random field theory. SurfStat. http://www.math. mcgill.ca/keith/surfstat. Accessed October 1, 2013

34. Halliday GM, Macdonald V, Henderson JM. A comparison of degeneration in the motor thalamus and cortex between progressive supranuclear palsy and Parkinson's disease. Brain 2005;128:2272-80

35. Schmahmann JD, Pandya DN. Disconnection syndromes of the basal ganglia, thalamus and cerebrocerebellar systems. Cortex 2008;44:1037-66

36. Whitwell JL, Avula R, Master A, et al. Disrupted thalamocortical connectivity in PSP: a resting state fMRI, DTI, VBM study. Parkinsonism Relat Disord 2011;17:599-605 